Orthopäde $2010 \cdot 39: 745$

DOI 10.1007/s00132-010-1621-5

Online publiziert: 28. Juli 2010

(c) Springer-Verlag 2010

\author{
C. Lüring · J. Grifka \\ Orthopädische Klinik, Universität Regensburg, Asklepios-Klinikum Bad Abbach
}

\title{
Klinische Behandlungspfade in Orthopädie und Traumatologie
}

\section{Ein Überblick}

Zeit ist Geld. Das ist eine altbekannte und viel zitierte Weisheit, die in den vergangenen Jahren zunehmend Gewicht in der Medizin erhält. Gerade im Krankenhaus werden aber oftmals sog. Blindleistungen ausgeführt, die keinen direkten Nutzen für den Behandlungserfolg des Patienten besitzen. Nicht alle, aber einige Aktivitäten, die nicht direkt der Behandlung dienen, können gestrafft oder verlassen werden. Doppelte Untersuchungen und Wartezeiten auf Untersuchungsergebnisse, Ärzte, Pflegekräfte und weitere Teammitarbeiter können sich ebenfalls negativ auf die Wertschöpfung einer Abteilung auswirken und die dort Wirkenden demotivieren.

\section{( Klinische Pfade verbessern die Wertschöpfung einer Abteilung und motivieren Mitarbeiter}

Implementiert man in einer Klinik für gut definierte Krankheitsbilder klinische Pfade („clinical pathways“), kann man die Wertschöpfung einer Abteilung verbessern, die Mitarbeiter motivieren und $\mathrm{Pa}$ tienten den Behandlungsablauf verdeutlichen. Aus dem Gesagten lässt sich zweifelsfrei ableiten, dass die gezielte Organisation einer Behandlung nicht nur sinnvoll sondern zwingend erforderlich ist. Auch unter den Gesichtspunkten des leitlinienkonformen Handelns und der Verpflichtung zur Einführung und Weiterentwicklung eines Qualitätsmanagementsystems ist die Implementierung von „clinical pathways" eine notwendige Investi- tion in die Verbesserung des klinischen Ablaufs.

Das vorliegende Themenheft soll über den betriebswirtschaftlichen und medizinischen Sinn von klinischen Pfaden berichten, anhand von Beispielen aufzeigen, wo Pfade gut und verlässlich einsetzbar sind und die Notwendigkeit des Benchmarkings herausstellen.
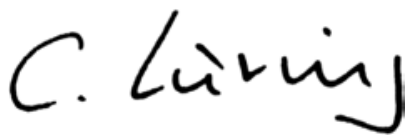

C. Lüring

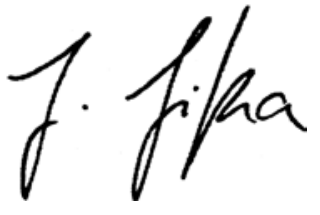

J. Grifka

\section{Korrespondenzadresse}

PD Dr. C. Lüring

Orthopädische Klinik, Universität Regensburg, Asklepios-Klinikum Bad Abbach Kaiser-Karl-Allee 3, 93077 Bad Abbach c_luering@yahoo.de 